\title{
Antagonismo in vitro de hongos endófitos para su uso en el biocontrol de enfermedades forestales
}

\author{
Juan Asdrúbal Flores-Pacheco ${ }^{1}$
}

\section{RESUMEN}

Los hongos endófitos, son aquellos que colonizan los tejidos vivos internos de las plantas sin causar efecto significativo en el hospedante. Estos hongos, al ejercer en ocasiones, antagonismo frente a patógenos fúngicos a la planta hospedante, pueden ser una solución para el control de muchas de las enfermedades forestales causadas por otros hongos. Este campo ha sido poco evaluado en pruebas in vitro y en pruebas en vivo. Se utilizaron cinco hongos endófitos y seis hongos patógenos que se enfrentaron en medio de cultivo generalista Papa + Dextrosa +Agar (PDA) en condiciones de oscuridad a $25^{\circ} \mathrm{C}$ por siete días. Durante este periodo se midieron los ejes centrales y laterales de la colonia. Con estos datos se obtuvo el coeficiente de forma del hongo y se calculó el crecimiento directo, el coeficiente de forma del hongo patógeno frente al endófito respectivo, el porcentaje de inhibición del crecimiento radial y el porcentaje de inhibición de la zona de crecimiento. Se establecieron placas únicamente con el hongo patógeno a manera de grupo control. Los datos se analizaron con un Análisis de Varianza y para los casos estadísticamente significativos se aplicó la prueba de Diferencia Mínima Significativa. Los resultados muestran que los endófitos que tienen una mayor inhibición frente a los hongos patógenos. De esta manera se demuestra un efecto controlador de al menos un endófito sobre uno o más patógenos. Esto sugiere su posible utilidad como Agente de Control Biológico (ACB) ante enfermedades forestales. La reducción del crecimiento de los hongos patógenos ante la presencia de los endófitos, puede deberse a los mecanismos de acción de éstos (competencia y secreción de micotoxinas) frente al patógeno. Se deben realizar ensayos in vivo donde los endófitos se inoculen de acuerdo con su ecología y con un tiempo adecuado para la colonización de la planta.

Palabras claves: control biológico, hongos patógenos, sanidad vegetal, antagonismo in vitro.

Recibido: 20 de junio de 2017

Aceptado: 27 de noviembre de 2017

1 Docente Investigador en la Facultad de Recursos Naturales y Medio Ambiente, Bluefields Indian \& Caribbean University- BICU, Apartado postal No 88, Avenida Universitaria, Bluefields, Nicaragua. Doctor en Conservación y Sistemas Forestales Sostenibles / Patología Forestal y Fisiología Vegetal. Correo electrónico: juan18asdrubal@gmail.com; japachecobicu@gmail.com 


\title{
In vitro antagonism of endophytic fungi for use in the biocontrol of forest diseases
}

\begin{abstract}
Endophytic fungi are those that colonize the internal living tissues of plants without causing significant effect on the host. These fungi, when exerting at times, antagonism against fungal pathogens to the host plant, can be a solution for controlling many of the forest diseases caused by other fungi. This field has been little evaluated in in vitro tests and in live tests. Five endophytic fungi and six pathogenic fungi were used, which were exposed in a generalized potato + Dextrose + Agar (PDA) culture medium under dark conditions at $25^{\circ} \mathrm{C}$ for seven days. During this period the central and lateral axes of the colony were measured. With these data, the shape coefficient of the fungus was obtained and the direct growth was calculated, the shape coefficient of the pathogenic fungus against the respective endophyte, the percentage of inhibition of radial growth and the percentage of inhibition of the growth zone. Plaques were established only with the pathogenic fungus as a control group. The data were analyzed with an Analysis of Variance and for the statistically significant cases the Significant Minimum Difference test was applied. The results show that endophytes have a greater inhibition against pathogenic fungi. In this way a controlling effect of at least one endophyte on one or more pathogens is demonstrated. This suggests its possible usefulness as a Biological Control Agent (CBA) in forest diseases. The reduction of the growth of pathogenic fungi in the presence of endophytes may be due to their mechanisms of action (competition and secretion of mycotoxins) against the pathogen. In vivo tests should be carried out where the endophytes are inoculated according to their ecology and with an adequate time for the colonization of the plant.
\end{abstract}

Keywords: biological control, pathogenic fungi, plant health, in vitro antagonism. 


\section{INTRODUCCIÓN}

Los hongos endófitos, se definen como aquellos que colonizan los tejidos vivos internos de las plantas sin causar un efecto significativo en el hospedante (Botella and Diez, 2011), A pesar de su escasa implantación como Agentes de Control Biológico (ACB) en campo, han sido ya utilizados con éxito para la protección de las plantas (Arnold et al., 2003). De este modo, una de las líneas de trabajo más importantes en el ámbito de la protección forestal frente a enfermedades consiste en el estudio de la micoflora interna de las especies vegetales.

En la actualidad, no se han encontrado medidas de control basadas en endófitos con aplicación en viveros y plantaciones forestales. Un enfoque de gestión integrada que permita la combinación de estrategias ecosostenibles, ambientalmente inocuos y económicamente rentables, que permitan reducir el impacto económico y ecológico de estas enfermedades. La gestión integrada puede incluir medidas de cuarentena, mejora genética y manejo silvicultural adecuado, así como la selección genética de clones de especies que son menos susceptibles a patógenos (Wingfield et al., 2008; Porter et al., 2009).

En esta clase de manejo integrada ha de tener también cabida el control biológico. Particularmente, el uso de hongos endófitos, (Martínez-Álvarez, et al., 2016), tiene un futuro prometedor tanto por los resultados que proporciona (Bailey and Gilligan, 1997) como por la inocuidad en el ecosistema en que se emplee. Hasta el momento el control biológico ha sido empleado con éxito en el control de varias patologías vegetales (Trestić et al., 2001; Kwon et al., 2009; Romeralo, et al., 2013; Martínez-Álvarez, 2015).

Las interacciones entre los hongos fitopatógenos y los hongos endófitos, son conocidas desde principios del siglo XX (Weindling, 1932), siendo ya desde entonces objeto de estudio el uso de este hongos endófitos como Agente de Control Biológico (ACB) (Mehrotra et al.,
1998, Yang, 1995). Actualmente se sabe que los tejidos vegetales que contienen hongos endófitos afectan la resistencia de sus hospedantes frente a parásitos (Miller, 1986; Vinale et al., 2008). Por ejemplo, varios estudios han demostrado que las plantas inoculadas con endófitos incrementan su crecimiento (Barka et al., 2002), mejoran la resistencia a la sequía (Swarthout et al., 2009), toleran condiciones de suelos no aptos (Malinowski et al., 2005), tienen mayor acceso a nutrientes (Robin et al., 2000) o mejoran la defensa frente a animales herbívoros (Bernstein and Carroll, 1977; Carrol, 2012) y patógenos (Arnold et al., 2003).

Este último beneficios, puede a su vez agruparse y diferenciarse en: efectos directos, indirectos y ecológicos (Gao et al., 2010). En los efectos directos los endófitos suprimen directamente al patógeno mediante la producción de antibióticos (Richardson et al., 2014) o la secreción de enzimas líticas (Vinale et al., 2008). Por otro lado, los efectos indirectos que los endófitos provocan en las plantas, ayudando a reducir el daño del patógeno pueden ser, por ejemplo, la inducción de la resistencia a la planta, la estimulación de producción de metabolitos y el incremento del crecimiento y de la actividad fisiológica. Por último, ejemplos de los efectos ecológicos son la ocupación de un nicho ecológico, el hiperparasitísmo y la depredación (Gao et al., 2010).

Uno de los endófitos más estudiados por su potencial como ACB en las enfermedades de las plantas es el género Trichoderma (Howell, 2003). Una de las características más destacadas que presenta este, es su habilidad para parasitar otros hongos (Weindling, 1932). Además este género es capaz de producir sustancias antibióticas que son inhibidoras de muchos patógenos (Juhásová et al., 2005), de las cuales destacan las enzimas chitinasas, gluconasas, así como el ácido 2.6 dichloroisonicotinico (Howell, 2003). Sin embargo, el principal mecanismo de control biológico de Trichoderma spp. es la competencia por el espacio y los nutrientes de la rizosfera (Howell, 2003). 
El crecimiento de este hongo, estudiado en este experimento, no está limitado al suelo o las raíces, sino que es capaz de colonizar el floema, xilema y médula de los árboles (Jankowiak, 2006). Existen muchos ejemplos de Trichoderma spp. y otros endófitos como controladores de enfermedades en plantas (LatundeDada, 1993; Maciá-Vicente et al., 2008; Ruano-Rosa et al., 2010) siendo en algunos casos enfermedades causadas por el género Fusarium (Sivan and Chet, 1987; Bernal-Vicente et al., 2009; Basak and Basak, 2011), y otros de interés forestal como Rosellinia necatrix (Ruano Rosa and López Herrera, 2009).

Los estudios realizados hasta el momento sobre el Control Biológico con hongos endófitos tal es el caso de patógenos como Fusarium circinatum, Phytophthora alni, P. pluvivora., Gremmeniella abietina, Cryphonectria parasitica y Heterobasidion annosum mediante el uso de hongos endófitos destacando el género Trichoderma spp. y Aspergillus. Se ha observado la interacción antagonista, por ejemplo, de Fusarium circinatum y las especies fúngicas Pinicillium chrysogenum Link y Fusarium lateritium Ness (Romón et al., 2008). Por último, también se ha evaluado el uso potencial de endófitos como Trichoderma spp y Clonostachys spp. como controladores de Fusarium circinatum, en plantas de Pinus radiata (Moraga-Suazo et al., 2011). Este estudio observó un incremento significativo de la supervivencia de las plantas de Pinus radiata con las cepas de Clonostachys spp probadas, no siendo así en el caso de los aislamientos de las cepas de Trichoderma spp.

En consecuencia, en el presente estudio se analizó el efecto antagonista de seis hongos endófitos de Pinus spp. F ente a seis patógenos forestales (Trichoderma atroviride, Mucor hiemalis, Chaetomium globosum, Aspergillus flavus, T. viride). Para ello se recurrió al análisis de diferentes variables coloniales a fin de determinar el grado de inhibición entre organismos.

\section{MATERIALES Y MÉTODOS}

\section{Efecto protector de hongos antagonistas contra la infección de patógenos forestales}

La evaluación del efecto antagonista de cada especie fúngica endófita (Tabla 1) contra cada especie patógena (Tabla 2) se realizó in vitro, mediante el emparejamiento de dobles aislados (patógeno - endófito). Los ensayos se llevaron a cabo en placas Petri de $90 \mathrm{~mm}$ de diámetro, utilizando medio de cultivo PDAs $3.90 \%$ patata-dextrosa-agar, suplementado con $0.50 \mathrm{mg} / 1 \mathrm{de}$ estreptomicina sulfato como agente antibiótico) sellado con papel Parafilm ${ }^{\circledR}$. Se cultivaron los organismos de forma aislada y se incubaron las placas a $25^{\circ} \mathrm{C}$ en la oscuridad durante una semana (Martínez-Álvarez et al., 2012).

Transcurrido este tiempo se extrajo un trozo de PDAS con micelio. Posteriormente, se tomaron placas Petricon PDA, en las cuales se sembraron los enfrentamientos de hongos patógenos contra hongos endófitos. Se utilizaron 5 repeticiones por cada enfrentamiento que consistió en la confrontación endófito vs patógeno. Esto se hizo colocando una sección de $5 \times 5 \mathrm{~mm}$ de micelio con medio PDAs con micelio de cada aislado en extremos opuestos de la placa, de forma tal, que se garantice el enfrentamiento de los micelios de las dos especies enfrentadas. La evaluación de la acción antagónica de cada especie se determinó por medio de la estimación del área de crecimiento micelial ocupada por cada especie. Se usaron cuatro indicadores para medir el efecto antagonista de los hongos endófitos sobre el patógeno (Martínez-Álvarez, 2016). Figura 1.

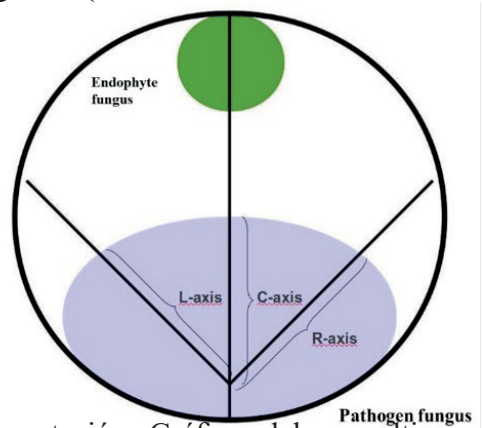

Figura 1. Representación Gráfica del co-cultivo entre patógenos forestales y hongos endófitos en el interior de una placa Petri (MartínezÁlvarez et al., 2016). 
Tabla 1. Hongos endófitos. Fuente de los aislados y códigos Martínez-Álvarez, P. et al., 2016.

\begin{tabular}{|c|c|c|c|c|c|c|c|c|}
\hline Especie & Código & $\begin{array}{l}\text { Año de } \\
\text { colecta }\end{array}$ & Hospedero & Localización & País & Latitud & Longitud & $\begin{array}{l}\mathbf{N}^{0} \text { Acceso } \\
\text { GenBank }\end{array}$ \\
\hline $\begin{array}{l}\text { Trichoderma } \\
\text { atroviride }\end{array}$ & HP136 & 2010 & Pinus radiata & $\begin{array}{l}\text { Comillas, } \\
\text { Cantabria }\end{array}$ & España & $43^{\circ} 20^{\prime} 16.2^{\prime \prime} \mathrm{N}$ & $4^{\circ} 18^{\prime} 17.1^{\prime \prime} \mathrm{W}$ & KT323338 \\
\hline Mucor hiemalis & HP139 & 2010 & Pinus radiata & $\begin{array}{l}\text { Comillas, } \\
\text { Cantabria }\end{array}$ & España & $43^{\circ} 20^{\prime} 16.2^{\prime \prime} \mathrm{N}$ & $4^{\circ} 18^{\prime} 17.1^{\prime \prime} \mathrm{W}$ & KT323340 \\
\hline $\begin{array}{l}\text { Chaetomium } \\
\text { globosum }\end{array}$ & HP148 & 2010 & Pinus radiata & $\begin{array}{l}\text { Comillas, } \\
\text { Cantabria }\end{array}$ & España & $43^{\circ} 20^{\prime} 16.2^{\prime \prime} \mathrm{N}$ & $4^{\circ} 18^{\prime} 17.1^{\prime \prime} \mathrm{W}$ & КT323349 \\
\hline Aspergillus flavus & HP149 & 2010 & Pinus radiata & $\begin{array}{l}\text { Comillas, } \\
\text { Cantabria }\end{array}$ & España & $43^{\circ} 20^{\prime} 16.2^{\prime \prime} \mathrm{N}$ & $4^{\circ} 18^{\prime} 17.1^{\prime \prime} \mathrm{W}$ & KT323350 \\
\hline Trichoderma viride & HP155 & 2010 & Pinus radiata & $\begin{array}{l}\text { Comillas, } \\
\text { Cantabria }\end{array}$ & España & $43^{\circ} 20^{\prime} 16.2^{\prime \prime} \mathrm{N}$ & $4^{\circ} 18^{\prime} 17.1^{\prime \prime} \mathrm{W}$ & KT323356 \\
\hline
\end{tabular}

\section{Variables Analizadas}

Las variables evaluadas en el presente ensayo se desglosan a continuación:

a. Longitud del eje central (eje C) de la colonia del hongo patógeno.

b. Coeficiente de la forma, calculado a partir de la diferencia del eje lateral y el eje central (Santamaría et al., 2007).

c. Porcentaje de inhibición del crecimiento radial $=$ 100 * ((r1-eje C))/r1 (ecuación 1), donde r1 es el máximo radio de la colonia del hongo patógeno en cualquier dirección en la placa control (Royse and Ries, 1978).

d. Porcentaje de inhibición de la zona de crecimiento. Para ello se utiliza la ecuación $\% \operatorname{Iz} \mathrm{C}=[(A-$
B)/A $]^{*} 100$ (ecuación 2 ), donde $\mathrm{A}=$ es el radio del hongo patógeno en la placa control $\mathrm{y}, \mathrm{B}=\mathrm{es}$ el radio del hongo patógeno en la placa de enfrentamiento (Orole and Adejumo, 2009).

\section{Análisis estadístico}

Los datos que se obtuvieron de este experimento fueron procesados en el programa estadístico SPSS (IBM $®$ Statistical SPSS $\AA$, 2016). Todas las variables cuantitativas fueron evaluadas para comprobar la normalidad y homogeneidad de varianzas, bajo este supuesto se aplicaron las pruebas de Análisis de Varianza (ANOVA) y Diferencia Mínima Significativa (DMS) de Fisher con $\alpha=0.05$, con el fin de identificar las diferencias en las variables evaluadas para cada tratamiento experimental.

Tabla 2. Patógenos forestales. Se numeran las referencias de los aislados de los patógenos utilizados MartínezÁlvarez, 2015, 2 Botella et al., 2010, 3-4 Centro de Sanidad Forestal de Calabazanos, 5-6 M. M. Haque, 2014.

\begin{tabular}{|c|c|c|c|c|c|c|c|c|}
\hline Especie & Código & $\begin{array}{l}\text { Año de } \\
\text { colecta }\end{array}$ & Hospedero & $\begin{array}{l}\text { Localiza- } \\
\text { ción }\end{array}$ & País & Latitud & Longitud & $\begin{array}{l}N^{0} \text { Acceso } \\
\text { GenBank }\end{array}$ \\
\hline $\begin{array}{l}\text { Fusarium } \\
\text { circinatum } 1\end{array}$ & FcCa6 & 2010 & $\begin{array}{l}\text { Pinus } \\
\text { radiata }\end{array}$ & $\begin{array}{l}\text { Comillas, } \\
\text { Cantabria }\end{array}$ & España & $\begin{array}{l}43^{\circ} 20^{\prime \prime} \\
16.20^{\prime} \mathrm{N}\end{array}$ & $\begin{array}{l}4^{\circ} 18^{\prime \prime} \\
17.10^{\prime} \mathrm{W}\end{array}$ & 041R01 \\
\hline $\begin{array}{l}\text { Heterobasidion } \\
\text { annosum } 2\end{array}$ & $\begin{array}{l}\text { A14009-AF- } \\
\text { ZAPR001 }\end{array}$ & 2014 & $\begin{array}{l}\text { Pinus } \\
\text { Pinaster }\end{array}$ & $\begin{array}{l}\text { Mahide, } \\
\text { Boya, } \\
\text { Zamora }\end{array}$ & España & $\begin{array}{l}41^{\circ} 52^{\prime \prime} \\
22.40^{\prime} \mathrm{N}\end{array}$ & $\begin{array}{l}6^{\circ} 22^{\prime \prime} \\
30.80^{\prime} \mathrm{W}\end{array}$ & ------------ \\
\hline $\begin{array}{l}\text { Gremmeniella } \\
\text { abietina } 3\end{array}$ & VAI-13 & 2003 & $\begin{array}{l}\text { Pinus } \\
\text { halepensis }\end{array}$ & $\begin{array}{l}\text { Villalba } \\
\text { de los } \\
\text { Alcores, } \\
\text { Palencia }\end{array}$ & España & $\begin{array}{l}31^{\circ} 43^{\prime \prime} \\
30.02^{\prime} \mathrm{N}\end{array}$ & $\begin{array}{l}2^{\circ} 54^{\prime \prime} \\
30.40^{\prime} \mathrm{W}\end{array}$ & FN663462 \\
\hline
\end{tabular}




\begin{tabular}{|c|c|c|c|c|c|c|c|c|}
\hline $\begin{array}{l}\text { Cryphonectria } \\
\text { parasitica } 4\end{array}$ & C1164-ZA28 & 2005 & $\begin{array}{l}\text { Castanea } \\
\text { sativa }\end{array}$ & $\begin{array}{l}\text { Arrabalde, } \\
\text { Zamora }\end{array}$ & España & $\begin{array}{l}26^{\circ} 03^{\prime \prime} \\
58.00^{\prime} \mathrm{N}\end{array}$ & $\begin{array}{l}46^{\circ} 65^{\prime \prime} \\
7.50^{\prime} \mathrm{W}\end{array}$ & ----------- \\
\hline $\begin{array}{l}\text { Phytophthora } \\
\text { alni } 5\end{array}$ & ZABRE02 & 2012 & $\begin{array}{l}\text { Alnus } \\
\text { glutinosa }\end{array}$ & $\begin{array}{l}\text { Betrocino, } \\
\text { Zamora }\end{array}$ & España & $\begin{array}{l}41^{\circ} 54^{\prime \prime} \\
48.00^{\prime} \mathrm{N}\end{array}$ & $\begin{array}{l}5^{\circ} 44^{\prime \prime} \\
45.60^{\prime} \mathrm{W}\end{array}$ & AF139366.1 \\
\hline $\begin{array}{l}\text { Phytophthora } \\
\text { pluvivora } 6\end{array}$ & SORLDD14 & 2010 & $\begin{array}{l}\text { Alnus } \\
\text { glutinosa }\end{array}$ & $\begin{array}{l}\text { Langa de } \\
\text { Duero, } \\
\text { Soria }\end{array}$ & España & $\begin{array}{l}41^{\circ} 36^{\prime \prime} \\
34.00^{\prime} \mathrm{N}\end{array}$ & $\begin{array}{l}3^{\circ} 25^{\prime \prime} \\
10.00^{\prime} \mathrm{W}\end{array}$ & KF413075 \\
\hline
\end{tabular}

\section{RESULTADOS}

Los cinco hongos endófitos evaluados en este ensayo (Tabla 1) empleados en este ensayo frente a distintos patógenos (Tabla 2), han sido seleccionados por su potencial como agentes de control biológico (ACB). Previamente fueron probados en ensayos in vivo (Martínez-Álvarez et al., 2016) demostrando por medio de la técnica del área bajo la curva (AUDPC) (Navarro Flores, 2016) carecen de potencial patogénico para especies forestales, específicamente para coníferas del género Pinus. En todos los casos se comparan los resultados del o de los hongos endófitos con mayor reducción del crecimiento del hongo patógeno con el control de este último.

Tal y como muestran las Figuras 2-5, presentan el efecto en la reducción del crecimiento $(\mathrm{mm})$ del eje $\mathrm{C}$ del patógeno frente a la acción del hongo endófito. Concretamente, la figura 2 muestra la reducción del crecimiento de la colonia de $F$. circinatum frente a Trichoderma viride que lo redujo un $82 \%$ (p-valor $<0.001$ ), mientras que, Trichoderma atroviride y Aspergillus flavus ambos generan un 65\% $(\mathrm{p}$-valor $<0.005)$ de reducción en el crecimiento de la colonia. Para este caso, Se nota cobertura total del área de la placa Petri por parte de los hongos endófito, incluso creciendo sobre la colonia de $F$. circinatum. En el caso de la figura 3, T. viride y $T$. atroviride que redujeron un $87.5 \%$ (p-valor $<0.001$ ) el crecimiento de Gremmeniella abietina.

En el caso de Heterobasidion annosum (figura 4) fue el endófito Mucor hiemalis el que causó la reducción del 84.21\% (p-valor $<0.001)$ frente al control. En este enfrentamiento se distinguen claramente las colonias de ambos hongos. Este mismo endófito redujo el crecimiento de Phytophthora plurivora (figura 7) en un $60.50 \%$ (p-valor $<0.05$ ), teniendo el mayor éxito de todos los enfrentamientos probados. Sin embargo, Aspergillus flavus presenta un potencial muy elevado como ACB. En el enfrentamiento con Cryphonectria parasitica (figura 5) redujo un $85.45 \%$ (p-valor $<0.001$ ) el crecimiento de este patógeno. Efecto que también genera en Phytophthora alni (figura 6) Phytophthora plurivora (figura 7) con 83.33\% (p-valor $<0.001$ ) y $68.41 \%$ (p-valor $<0.05)$, respectivamente.

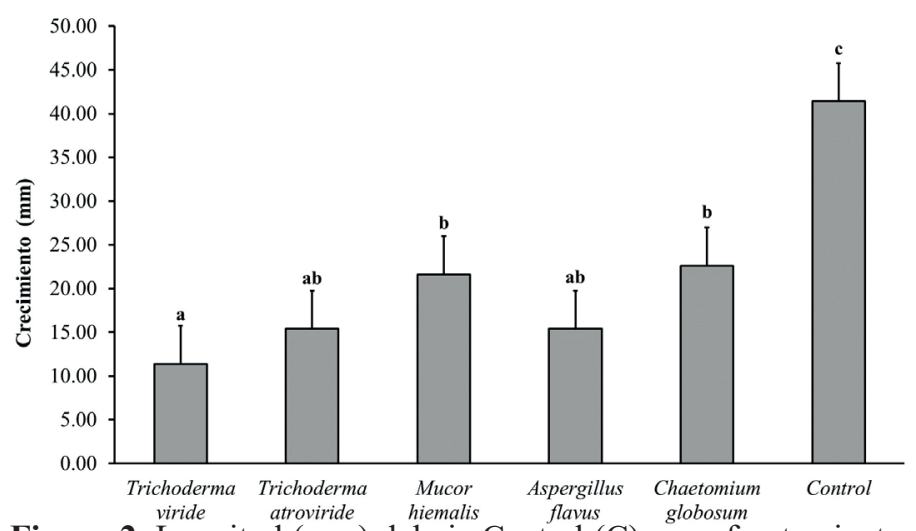

Figura 2. Longitud ( $\mathrm{mm}$ ) del eje Central (C) en enfrentamientos in vitro de Fusarium circinatum contra hongos endófitos. Prueba estadística Análisis de Varianza (ANOVA) - Diferencia Mínima Significativa 


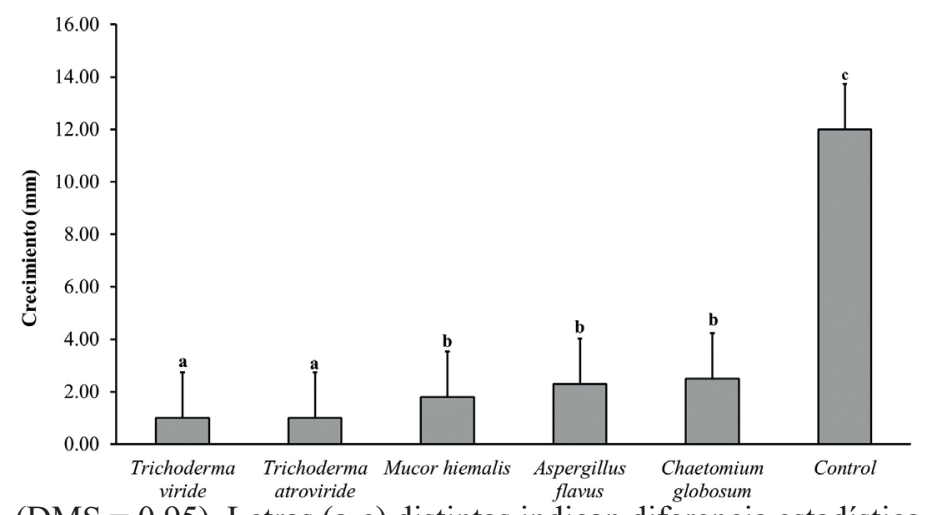

$(\mathrm{DMS}=0.95)$. Letras $(\mathrm{a}-\mathrm{c})$ distintas indican diferencia estadística $\alpha=0.05$. En la gráfica se han reflejado los valores de la Media (Md) y Desviación Estándar (DE).

Figura 3. Longitud (mm) del eje Central (C) en enfrentamientos in vitro de Gremmeniella abietina contra hongos endófitos. Prueba estadística Análisis de Varianza (ANOVA) - Diferencia Mínima Significativa (DMS $=0.95$ Letras $(\mathrm{a}-\mathrm{c})$ distintas indican diferencia estadística $\alpha=0.05$. En la gráfica se han reflejado los valores de la Media (Md) y Desviación Estándar (DE).

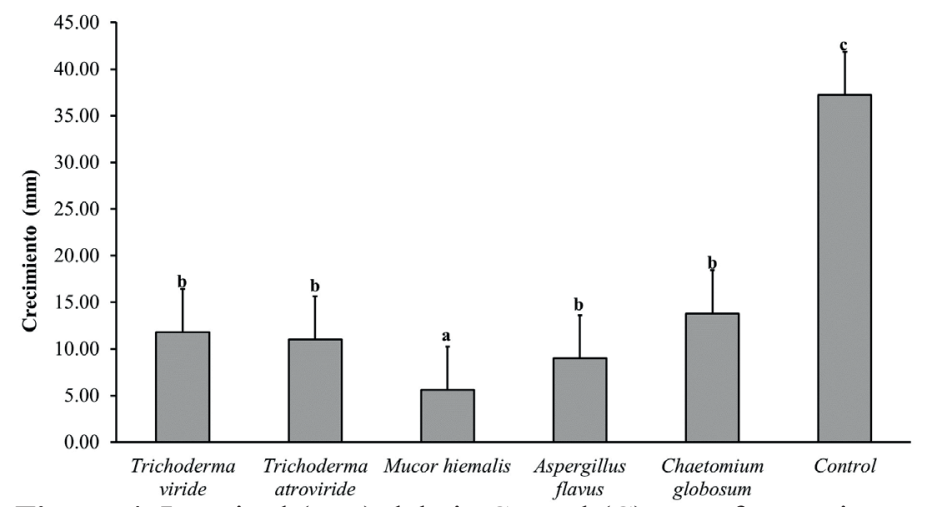

Figura 4. Longitud ( $\mathrm{mm}$ ) del eje Central (C) en enfrentamientos in vitro de Heterobasidion annosum contra hongos endófitos. Prueba estadística Análisis de Varianza (ANOVA) - Diferencia Mínima Significativa (DMS =0.95). Letras (a-c) distintas indican diferencia estadística $\alpha=0.05$. En la gráfica se han reflejado los valores de la Media (Md) y Desviación Estándar (DE).

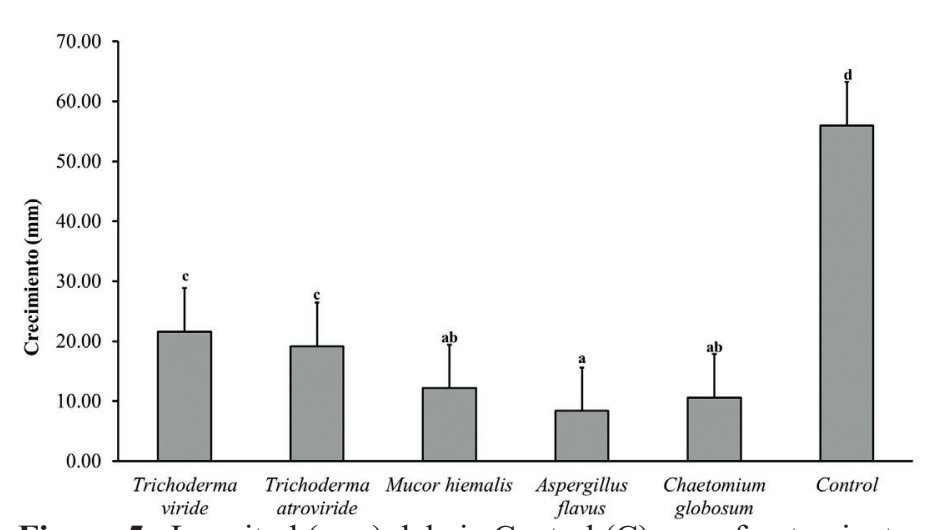

Figura 5. Longitud ( $\mathrm{mm}$ ) del eje Central (C) en enfrentamientos in vitro de Cryphonectria parasitica contra hongos endófitos. Prueba estadística Análisis de Varianza (ANOVA) - Diferencia Mínima Significativa (DMS $=0.95)$. Letras $(\mathrm{a}-\mathrm{c})$ distintas indican diferencia estadística $\alpha=0.05$. En la gráfica se han reflejado los valores de la Media (Md) y Desviación Estándar (DE).

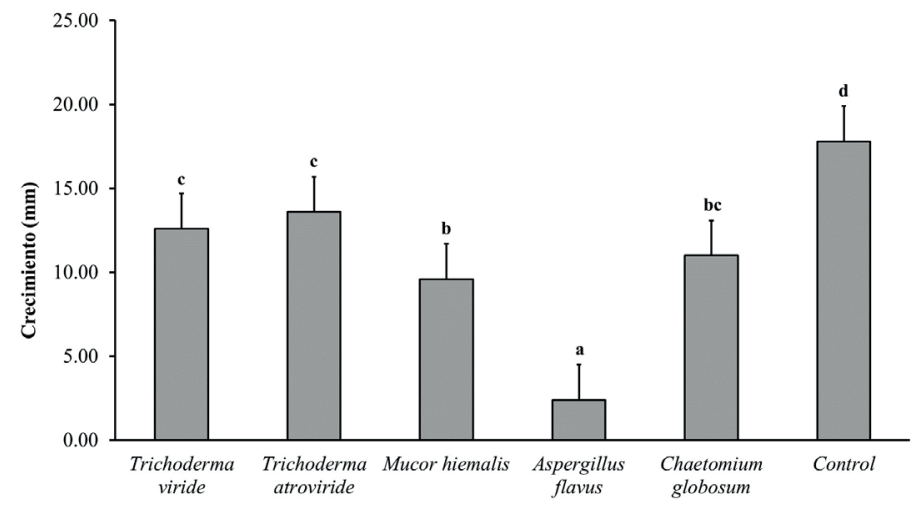

Figura 6. Longitud ( $\mathrm{mm}$ ) del eje Central (C) en enfrentamientos in vitro de Phytophthora alni contra hongos endófitos. Prueba estadística Análisis de Varianza (ANOVA) - Diferencia Mínima Significativa $(\mathrm{DMS}=0.95$. Letras $(\mathrm{a}-\mathrm{c})$ distintas indican diferencia estadística $\alpha=0.05$. En la gráfica se han reflejado los valores de la Media (Md) y Desviación Estándar (DE).

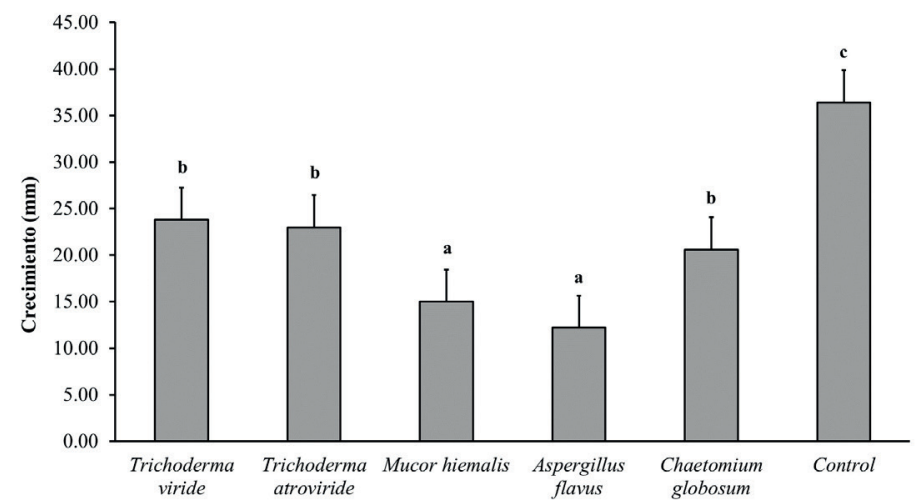

Figura 7. Longitud ( $\mathrm{mm}$ ) del eje Central (C) en enfrentamientos in vitro de Phytophthora plurivora contra hongos endófitos. Prueba estadística Análisis de Varianza (ANOVA) - Diferencia Mínima Significativa (DMS $=0.95)$. Letras $(\mathrm{a}-\mathrm{c})$ distintas indican 
diferencia estadística $\alpha=0.05$. En la gráfica se han reflejado los valores de la Media (Md) y Desviación Estándar (DE).

En la Tabla 3 muestran los tres parámetros obtenidos para estimar el efecto inhibidor de los hongos endófitos enfrentados a patógenos. Los parámetros contenidos son i) Coeficiente de la forma del patógeno, ii) Porcentaje de inhibición del crecimiento radical y iii) Porcentaje de inhibición de la zona de crecimiento. En el caso de $T$. viride y $T$. atroviride se encontraron diferencias significativas con un p-valor $<0.001$ en los tres parámetros monitoreados en los patógenos $F$. circinatum y $G$. abietina. Para P. alni únicamente $T$. viride mostró diferencias significativas ( $\mathrm{p}$-valor $<0.001$ ), aunque no se desprecia el $\mathrm{p}$-valor $<0.05 T$. atroviride sobre el mismo hongo. Al igual que en las figuras iniciales $M$. hiemalis mostro diferencia significativa ( $p$-valor $<0.001)$ en la inhibición del desarrollo de $H$. annosum y $P$. pluvivora, incluso estadísticamente superior $(\mathrm{p}$-valor $<0.05)$ a los valores registrados por las especies de Trichoderma. También, A. flavus mostro diferencia significativa sobre $C$. parasitica y $P$. alni (p-valor $<0.001$ ), en ambos casos, pero siendo el único con este grado de control para $C$. parasitica.

Tabla 3. Comparaciones múltiples para enfrentamientos in vitro de patógenos forestales y hongos endófitos. Md $=$ Media aritmética. $\mathrm{DE}=$ Desviación Estándar. Sig. = Indicador de diferencia significativa. * P-valor $<0.05$. ** $\mathrm{P}$-valor $<0.001 . \mathrm{ns}=$ sin diferencia significativa. Diferencia Mínima Significativa $(\mathrm{DMS}=0.95)$.

\begin{tabular}{|c|c|c|c|c|c|c|c|c|c|c|}
\hline \multirow[t]{2}{*}{$\begin{array}{l}\text { Especies pató- } \\
\text { genas }\end{array}$} & \multirow[t]{2}{*}{ Hongos endófitos } & \multicolumn{3}{|c|}{$\begin{array}{c}\text { Coeficiente de la forma } \\
\text { del patógeno }\end{array}$} & \multicolumn{3}{|c|}{$\begin{array}{l}\text { Porcentaje de inhibición } \\
\text { del crecimiento radial }\end{array}$} & \multicolumn{3}{|c|}{$\begin{array}{l}\text { Porcentaje de inhibición } \\
\text { de la zona de crecimiento }\end{array}$} \\
\hline & & Md & DE & Sig. & Md & DE & Sig. & Md & DE & Sig. \\
\hline \multirow{5}{*}{$\begin{array}{l}\text { Fusarium } \\
\text { circinatum }\end{array}$} & Trichoderma viride & 1.00 & 0.71 & ** & 75.74 & 4.15 & ** & 72.46 & 4.71 & $* *$ \\
\hline & Trichoderma atroviride & 1.40 & 0.89 & ** & 67.24 & 5.55 & ** & 62.80 & 6.30 & ** \\
\hline & Mucor hiemalis & 2.40 & 3.05 & * & 54.04 & 3.56 & * & 47.83 & 4.05 & * \\
\hline & Aspergillus flavus & 2.40 & 0.55 & * & 52.55 & 1.17 & * & 57.49 & 1.33 & * \\
\hline & Chaetomium globosum & 1.00 & 0.00 & ns & 51.91 & 2.43 & ns & 45.41 & 2.76 & ns \\
\hline \multirow{5}{*}{$\begin{array}{l}\text { Heterobasidion } \\
\text { annosum }\end{array}$} & Trichoderma viride & 1.20 & 0.45 & * & 71.22 & 4.01 & * & 68.28 & 4.42 & * \\
\hline & Trichoderma atroviride & 1.60 & 1.14 & * & 73.17 & 7.72 & * & 70.43 & 8.50 & * \\
\hline & Mucor hiemalis & 1.00 & 0.00 & ** & 86.34 & 3.70 & ** & 84.95 & 4.08 & $* *$ \\
\hline & Aspergillus flavus & 1.20 & 1.30 & * & 78.05 & 11.82 & * & 75.81 & 13.03 & * \\
\hline & Chaetomium globosum & 1.60 & 0.55 & ns & 66.34 & 4.01 & ns & 62.90 & 4.41 & * \\
\hline \multirow{5}{*}{$\begin{array}{l}\text { Gremmeniella } \\
\text { abietina }\end{array}$} & Trichoderma viride & 0.00 & 0.00 & ** & 83.05 & 0.00 & ** & 85.00 & 20.87 & $* *$ \\
\hline & Trichoderma atroviride & 0.00 & 0.00 & $* *$ & 83.05 & 0.00 & $* *$ & 85.00 & 0.00 & $* *$ \\
\hline & Mucor hiemalis & 0.00 & 0.00 & * & 61.02 & 0.00 & * & 65.00 & 0.00 & * \\
\hline & Aspergillus flavus & 0.00 & 0.00 & * & 55.93 & 0.00 & * & 59.33 & 0.00 & * \\
\hline & Chaetomium globosum & 0.00 & 0.00 & * & 68.81 & 1.52 & * & 50.00 & 2.24 & * \\
\hline \multirow{5}{*}{$\begin{array}{l}\text { Cryphonectria } \\
\text { parasitica }\end{array}$} & Trichoderma viride & 2.60 & 1.52 & * & 62.11 & 14.71 & * & 36.31 & 6.17 & * \\
\hline & Trichoderma atroviride & 3.20 & 2.49 & * & 66.32 & 16.89 & * & 38.25 & 20.32 & * \\
\hline & Mucor hiemalis & 2.60 & 3.65 & * & 68.59 & 13.27 & $* *$ & 47.38 & 24.91 & ** \\
\hline & Aspergillus flavus & 1.80 & 0.74 & $* *$ & 85.26 & 7.50 & $* *$ & 79.22 & 20.77 & ** \\
\hline & Chaetomium globosum & 2.00 & 1.00 & ns & 61.40 & 15.22 & ns & 59.03 & 19.09 & ns \\
\hline
\end{tabular}




\begin{tabular}{|c|c|c|c|c|c|c|c|c|c|c|}
\hline \multirow{5}{*}{$\begin{array}{l}\text { Phytophthora } \\
\text { alni }\end{array}$} & Trichoderma viride & 1.40 & 1.14 & ** & 52.00 & 5.70 & * & 46.21 & 6.40 & * \\
\hline & Trichoderma atroviride & 0.60 & 0.55 & * & 32.00 & 4.47 & $* *$ & 23.60 & 5.02 & * \\
\hline & Mucor hiemalis & 1.00 & 0.71 & ns & 37.00 & 10.37 & ns & 21.60 & 11.65 & ns \\
\hline & Aspergillus flavus & 0.60 & 1.34 & ** & 88.00 & 26.83 & ** & 86.52 & 30.15 & $* *$ \\
\hline & Chaetomium globosum & 0.60 & 0.89 & * & 45.00 & 8.66 & * & 38.20 & 9.73 & * \\
\hline \multirow[t]{5}{*}{ P. pluvivora } & Trichoderma viride & 3.80 & 1.30 & * & 40.50 & 5.70 & * & 34.62 & 6.26 & ** \\
\hline & Trichoderma atroviride & 1.60 & 0.89 & * & 42.50 & 7.91 & * & 36.81 & 8.69 & * \\
\hline & Mucor hiemalis & 1.40 & 1.14 & ** & 62.50 & 3.95 & $* *$ & 58.79 & 4.34 & $* *$ \\
\hline & Aspergillus flavus & 2.00 & 1.41 & * & 39.50 & 15.55 & ** & 36.48 & 17.09 & * \\
\hline & Chaetomium globosum & 2.40 & 0.89 & * & 48.50 & 5.76 & * & 43.41 & 6.33 & * \\
\hline
\end{tabular}

\section{DISCUSIÓ $\mathbf{N}$}

Los organismos patógenos evaluados en el presente estudio, generan graves daños ecológicos y económicos a escala global por lo que han sido catalogados como de interés por la Organización europea y mediterránea de protección fitosanitaria (EPPO) (2006a). Principalmente en los países donde se hallan detectados estos patógenos se han llevado investigaciones para su control. Sin embargo, muchas de ellas se han dirigido a la búsqueda de resistencia del hospedante, son muy pocas las que exploran el control biológico por medio de hongos endófitos (Zabalgogeazcoa, 2008, Abello and Kelemu, 2006).

De acuerdo a los trabajos publicados por MartínezÁlvarez et al., (2012, 2016) las especies de Trichoderma spp., frente a F. circinatum. Demostró un elevado efecto de control inhibitorio por producción de micotoxinas de carácter antifúngicas combinadas con su capacidad de micoparasitar al huésped (Rolando et al., 2013). Estas micotoxinas inhibitorias actuaron tanto sobre $F$. circinatum evitando el crecimiento de la colonia (Sánchez-Fernández et al., 2013), pero en otros patógenos como es el caso de G. abietina y P. alni no solo redujeron el crecimiento de la colonia, sino que también parasitó la colonia del patógeno al punto de cubrir totalmente la placa Petri.

Las dos especies de Trichoderma es el hongo endófito que tiene mejor efecto sobre $F$. circinatum y $G$. abietina (Romeralo et al., 2015). Sin embargo, el éxito de este endófito está relacionado directamente con la zona y momento de inoculación, incluso siendo más determinante que la concentración utilizada (Orole and Adejumo, 2009). De acuerdo a publicaciones de Moraga-Suazo et al., (2014) al realizar un estudio en el que se probaron varias cepas de Trichoderma spp. y de Clonostachys spp., para el control de F. circinatum no se obtuvieron resultados positivos al inocular el endófito en el tejido vegetal o en el sustrato al tiempo o luego que el patógeno. Contrariamente, en los casos en los que el patógeno fue inoculado, bien fuera en tejido vegetal o sustrato, posteriormente a la introducción del endófito, se observó un claro efecto antagónico de este último (Aleandri et al., 2015).

El género Trichoderma posee diferentes mecanismos de acción que pueden favorecer a la planta hospedante ante la presencia de un fitopatógeno (Verma et al., 2007), entre los que cabe destacar la competencia y la antibiosis (Sánchez-Fernández et al., 2013). La competencia en Trichoderma spp. es muy alta ya que biológicamente tiende a colonizar agresivamente el sustrato y a sobrevivir en casos de carencia de sustrato en forma de clamidosporas, además de que puede crecer sobre distintos sustratos y tiene una elevada tasa de esporulación (Bailey et al., 2008). La antibiosis es el mecanismo que permite a Trichoderma spp inhibir a los patógenos a partir de la producción de sustancias inhibidoras consideradas antibióticos y micotoxinas. Para el caso del endófito $C$. globosum el principal mecanismo de acción es la micostática, mejor conocido como efecto fungistático, que consiste en inhibir 
el crecimiento de otros hongos, pero sin provocar la muerte de antagonista (Rolando et al., 2013). Aspergillus flavus compite directamente por espacio, nutrientes y recursos necesarios para el desarrollo del micelio e hifas de ambos hongos (Bettiol, et al., 2014).

El hongo endófito, A. flavus, presentó mayor efecto inhibitorio frente a los patógenos $C$. parasítica, $P$. alni y P. pluvivora en concordancia con lo encontrado por Robin et al., (2000). Además, mostró gran diferencia con el resto de endófitos en estos dos últimos patógenos. Aunque no es de gran relevancia ya que es bastante conocida por sus efectos positivos ante muchos patógenos $\mathrm{y}$ ha sido objeto de estudio en muchas ocasiones como agente de control biológico (ACB). Mucor hiemalis que tuvo efecto frente a $H$. annosum en cambio en el resto de patógenos no redujo su crecimiento. Estudios de Yakimenko and Grodnitskaya, (2000) indican que al integrar este endófito en el suelo se registró la reducción la incidencia de enfermedades en plántulas de coníferas. Consideran que este hecho se debe a la mejora y aumento de la microflora del suelo y de la planta que aumento la capacidad de la planta para resistir infección de patógenos.

\section{CONCLUSIONES}

Es necesario continuar con la siguiente fase, la prueba in vivo de los cinco endófitos frente a los respectivos patógenos. Debe prestarse especial atención a la forma y tiempos de inoculación de los endófitos (Jankowiak, 2006) para obtener el máximo de su potencial colonizador y protector-estimulante (Ruppel et al., 1983). Es recomendable la producción de plantas con semillas y sustrato estéril en el que los endófitos sean inoculados al suelo al momento de la siembra de la semilla (Martínez-Álvarez et al., 2012). En el caso de utilizar plantas provenientes de vivero se recomienda la misma vía de inoculación del endófito con al menos 15 a 42 días de antelación a la inoculación del patógeno respectivo (Aleandri et al., 2015).
Teniendo en cuenta los resultados obtenidos en el presenten estudio, es prometedor el potencial de estos hongos endófitos como Agentes de Control Biológico de hongos patógenos de amplia distribución mundial. Deben de estudiarse con mayor profundidad, en pruebas de patogenicidad in vivo, la acción de las cepas de Trichoderma spp. sobre $F$. circinatum y $G$. abietina, así como $M$. hiemalis sobre $H$. annosum y $P$. plurivora. También deben tomarse en consideración los resultados positivos que se obtuvieron con $A$. flavus sobre $C$. parasitica y $P$. alni. En consecuencia, se ha determinado el efecto antagonista in vitro de cada uno de los endófitos frente a los patógenos a los que se enfrentaron, esto abre confirma su potencial para el control biológico. Esto debe ser confirmado con más pruebas y ensayo in vivo de los endófitos inoculados en los hospederos de cada patógeno.

\section{REFERENCIAS BIBLIOGRÁFICAS}

ABELLO, J.F., and S. KELEMU. 2006. Hongos endófitos: ventajas adaptativas que habitan en el interior de las plantas. Revista Corpioca - Ciencia y Tecnología Agropecuaria 7: 55-57.

ALEANDRI, M.P., G. CHILOSI, N. BRUNI, A. TOMASSINI, A.M. VETTRAINO, and A. VANNINI. 2015. Use of nursery potting mixes amended with local Trichoderma strains with multiple complementary mechanisms to control soil-borne diseases. Crop Protection 67: 269278. Available at: http://dx.doi.org/10.1016/j. cropro.2014.10.023.

ARNOLD, A.E., L.C. MEJÍA, D. KYLLO, E.I. ROJAS, Z. MAYNARD, N. ROBBINS, and E.A. HERRE. 2003. Fungal endophytes limit pathogen damage in a tropical tree. Proceedings of the National Academy of Sciences of the United States of America 100: 15649-15654.

BAILEY, B.A., H. BAE, M.D. STREM, J. CROZIER, S.E. THOMAS, G.J. SAMUELS, B.T. VINYARD, and K.A. HOLMES. 2008. Antibiosis, mycoparasitism, and colonization success for 
endophytic Trichoderma isolates with biological control potential in Theobroma cacao. Biological Control 46: 24-35.

BAILEY, D.J., and C.A. GILLIGAN. 1997. Biological control of pathozone behaviour and disease dynamics of Rhizoctonia solani by Trichoderma viride. New Phytologist 136: 359-367.

BARKA, E.A., S. GOGNIES, J. NOWAK, J.C. AUDRAN, and A. BELARBI. 2002. Inhibitory effect of endophyte bacteria on Botrytis cinerea and its influence to promote the grapevine growth. Biological Control 24: 135-142.

BASAK, A.C., and S.R. BASAK. 2011. Biological control of Fusarium solani sp. dalbergiae, the wilt pathogen of dalbergia sissoo, by Trichoderma viride and T. harzianum. Journal of Tropical Forest Science 23: 460-466.

BERNAL-VICENTE,A., M. ROS, and J.A.PASCUAL. 2009. Increased effectiveness of the Trichoderma harzianum isolate T-78 against Fusarium wilt on melon plants under nursery conditions. Journal of the Science of Food and Agriculture 89: 827-833.

BERNSTEIN, M.E., and G.C. CARROLL. 1977. Microbial populations on Douglas fir needle surfaces. Microbial Ecology 4: 41-52.

BOTELLA, L., and J. JAVIER DIEZ. 2011. Phylogenic diversity of fungal endophytes in Spanish stands of Pinus halepensis. Fungal Diversity 47: 9-18.

BOTELLA, L., T.T. TUOMIVIRTA, J. KAITERA, V. CARRASCO NAVARRO, J.J. DIEZ, and J. HANTULA. 2010. Spanish population of Gremmeniella abietina is genetically unique but related to type A in Europe. Fungal Biology 114: 778-789.

BUENO, A., J.J. DIEZ, and M.M. FERNÁNDEZ. 2010. Ophiostomatoid fungi transported by Ips sexdentatus (Coleoptera; Scolytidae) in Pinus pinaster in NW Spain. Silva Fennica 44: 387-397.

CARROL, G. 2012. Fungal Endophytes in Stems and Leaves: From Latent Pathogen to Mutualistic Symbiont. Ecology 69: 2-9.
CENTRO DE SANIDAD FORESTAL DE CALABAZANOS. 2007. Junta de Castilla y León. Polígono Industrial de Villamuriel S/N, 34190 Villamuriel de Cerrato, Palencia, Spain0-1.

EPPO. 2006. First report of Gibberella circinata in France. European and Mediterranean Plant Protection Organization 104: 9.

GAO, F.K., C.C. DAI, and X.Z. LIU. 2010. Mechanisms of fungal endophytes in plant protection against pathogens. African Journal of Microbiology Research 4: 1346-1351.

HOWELL, C.R. 2003. A case in point is that of T. virens-treated root segments taken from soil heavily infested with propagules of Macrophomina phaseo- lina, the pathogen that causes charcoal rot in a wide range of crops Biological Control of Plant Diseases: the history and . Plant Disease 87: 4-10. IBM ${ }^{\circledR}$ STATISTICAL SPSS ${ }^{\circledR}$. 2016. IBM ${ }^{\circledR}$ SPSS ${ }^{\circledR}$ 24.0. Statistical Package for the Social Sciences. Available at: http://www.spss.com/.

JANKOWIAK, R. 2006. Fungi associated with Tomicus piniperda in Poland and assessment of their virulence using Scots pine seedlings. Annals of Forest Science 63: 801-808.

JUHÁSOVÁ, G., K. ADAMCÍKOVÁ, and C. ROBIN. 2005. Results of biological control of chestnut blight in Slovakia. Phytoprotection 86: 19-23.

KWON, S.-J.J., W.-S.S. LIM, S.-H.H. PARK, M.R.R. PARK, and K.-H.H. KIM. 2009. Erratum: Molecular characterization of a dsRNA mycovirus, Fusarium graminearum Virus-DK21, which is phylogenetically related to hypoviruses but has a genome organization and gene expression strategy resembling those of plant potex-like viruses (Molecules. Molecules and Cells 28: 73-74.

LATUNDE-DADA, A.O. 1993. Biological-Control of Southern Blight Disease of Tomato Caused by Sclerotium rolfsii with Simplified Mycelial Formulations of Trichoderma koningii. Plant Pathology 42: 522-529.

MACIÁ-VICENTE, J.G., H.B. JANSSON, S.K. ABDULLAH, E. DESCALS, J. SALINAS, and L. 
V.LOPEZ-LLORCA.2008. Fungal rootendophytes from natural vegetation in Mediterranean environments with special reference to Fusarium spp. FEMS Microbiology Ecology 64: 90-105.

MALINOWSKI, D.P., H. ZUO, D.P. BELESKY, and G.A. ALLOUSH. 2005. Evidence for copper binding by extracellular root exudates of tall fescue but not perennial ryegrass infected with Neotyphodium spp. endophytes. Plant and Soil 267: 1-12.

MARTÍNEZ-ÁLVAREZ, P. 2015. Environmentally friendly methods for the integrated management of pine pitch canker (PPC) disease. University of Valladolid - INIA.

MARTÍNEZ-ÁLVAREZ, P., F.M. ALVES-SANTOS, and J.J. DIEZ. 2012. In vitro and in vivo interactions between Trichoderma viride and Fusarium circinatum. Silva Fennica 46: 303-316.

MARTÍNEZ-ÁLVAREZ， P., R.A. FERNÁNDEZGONZÁLEZ, A.V. SANZ-ROS, V. PANDO, and J.J. DIEZ. 2016. Two fungal endophytes reduce the severity of pitch canker disease in Pinus radiata seedlings. Biological Control 94: 1-10.

MEHROTRA, A. K., G. A., and A. A. 1998. Fungiagents of biological control in biocontrol of plant diseases. Mehrotra R., Aneja K., Gupta A., A. A. Boca Ratón: C.R.C. Press.

MILLER, J.D. 1986. Toxic metabolites of epiphytic and endophytic fungi of conifer needles. In $F$. \& Van den Heuvel Microbiology of the phyllosphere. Cambridge University Press 1: .

MORAGA-SUAZO, P., A. OPAZO, S. ZALDÚA, G. GONZÁLEZ, and E. SANFUENTES. 2011. Evaluation of Trichoderma spp. and Clonostachys spp. Strains to Control Fusarium circinatum in Pinus radiata Seedlings. Chilean journal of agricultural research 71: 412-417.

MORAGA-SUAZO, P., L. ORELLANA, P. QUIROGA, C. BALOCCHI, E. SANFUENTES, R.W. WHETTEN, R. HASBÚN, and S. VALENZUELA. 2014. Development of a genetic linkage map for Pinus radiata and detection of pitch canker disease resistance associated QTLs. Trees - Structure and Function 28: 1823-1835.

NAVARRO FLORES, J.R. 2016. La técnica del área bajo la curva. Centro de Investigaciones en Protección de Cultivos Universidad de Costa Rica. OROLE, O.O., and T.O. ADEJUMO. 2009. Activity of fungal endophytes against four maize wilt pathogens. African Journal of Microbiology Research 3: 969-973.

PORTER, B., M.J. WINGFIELD, and T.A. COUTINHO. 2009. Susceptibility of South African native conifers to the pitch canker pathogen, Fusarium circinatum. South African Journal of Botany 75: 380-382.

RICHARDSON, S.N., A.K. WALKER, T.K. NSIAMA, J. MCFARLANE, M.W. SUMARAH, A. IBRAHIM, and J.D. MILLER. 2014. Griseofulvinproducing Xylaria endophytes of Pinus strobus and Vaccinium angustifolium: Evidence for a coniferunderstory species endophyte ecology. Fungal Ecology 11: 107-113.

ROBIN, C., C. ANZIANI, and P. CORTESI. 2000. Relationship between biological control, incidence of hypovirulence, and diversity of vegetative compatibility types of Cryphonectria parasitica in France. Phytopathology 90: 730-737.

ROLANDO, Á., R. CARRIÓN1, R. NINA, H. SMELTEKOP, J. ALMANZA, M. LOZAMURGUIA, A.C. MICHEL-ACEVES, ET AL. 2013. Eficiencia biológica de cepas nativas de Trichoderma spp., en el control de Sclerotium rolfsii Sacc., en cacahuate. Avances de Investigación Agropecuaria 28: 89-107.

ROMERALO, C., L. BOTELLA, O. SANTAMARIA, and J.J. DIEZ. 2013. Control biológico de Gremmeniella abietina en plántulas de Pinus halepensis en invernadero mediante el uso de hongos endófitos. Congreso Forestal Espanol 6CFE01-383: 1-11.

ROMERALO, C., and R. ROMERALO-TAPIA. 2015. Management of Gremmeniella abietina by means 
of biological control agents and host resistance. Universidad de Valladolid. España190.

ROMERALO, C., J. WITZELL, R. ROMERALOTAPIA, L. BOTELLA, and J.J. DIEZ. 2015. Antagonistic activity of fungal endophyte filtrates against Gremmeniella abietina infections on Aleppo pine seedlings. European Journal of Plant Pathology 143: 691-704.

ROMÓN, P., M. TROYA, M.E.F. DE GAMARRA, A. EGUZKITZA, J.C.C. ITURRONDOBEITIA, A. GOLDARAZENA, M.E. FERNÁNDEZ DE GAMARRA, ET AL. 2008. Fungal communities associated with pitch canker disease of Pinus radiata caused by Fusarium circinatum in northern Spain: association with insects and pathogensaprophyte antagonistic interactions. Canadian Journal of Plant Pathology-Revue Canadienne De Phytopathologie 30: 241-253.

ROYSE, D.J., and S.M. RIES. 1978. The Influence of Fungi Isolated from Peach Twigs on the Pathogenicity of Cytospora cincta. Phytopathology 68: 603-607.

RUANO-ROSA, D., L. DEL MORAL-NAVARRETE, C.J. LOPEZ-HERRERA, and L. DEL MORALNAVARRETE. 2010. Selection of Trichoderma spp . isolates antagonistic to Rosellinia necatrix. Spanish Journal of Agricultural Research 8: 10841097.

RUANO ROSA, D., and C.J. LÓPEZ HERRERA. 2009. Evaluation of Trichoderma spp. as biocontrol agents against avocado white root rot. Biological Control 51: 66-71.

RUPPEL, E.G., R. BAKER, G.E. HARMAN, J.P. HUBBARD, R.J. HECKER, and I. CHET. 1983. Field tests of Trichoderma harzianum Rifai aggr. as a biocontrol agent of seedling disease in several crops and Rhizoctonia root rot of sugar beet. Crop Protection 2: 399-408.

SÁNCHEZ-FERNÁNDEZ， R.， B. SÁNCHEZORTIZ, Y. SANDOVAL-ESPINOSA, A. ULLOABENÍTEZ., B. ARMENDÁRIZ-GUILLÉN., M. GARCÍA-MÉNDEZ., and M. MACÍAS-
RUBALCAVA. 2013. Hongos endófitos: fuente potencial de metabolitos secundarios bioactivos con utilidad en agricultura y medicina. Tip 16: 132-146.

SANTAMARÍA, O, L. BOTELLA, and J. DIEZ. 2007. Gremmeniella abietina in North-western Spain: Distribution and associated mycoflora. Acta Silvatica and Lignaria Hungarica137-145.

SIVAN, A, and I. CHET. 1987. Biological control of Fusarium crown rot of tomato by Trichoderma hazianum under field condition. Plant Disease 71: 587-592.

SWARTHOUT, D., E. HARPER, S. JUDD, D. GONTHIER, R. SHYNE, T. STOWE, and T. BULTMAN. 2009. Measures of leaf-level wateruse efficiency in drought stressed endophyte infected and non-infected tall fescue grasses. Environmental and Experimental Botany 66: 8893.

TRESTIĆ, T., M. UŠČUPLIĆ, C. COLINAS, G. ROLLAND, A. GIRAUD, and C. ROBIN. 2001. Vegetative compatibility type diversity of Cryphonectria parasitica populations in BosniaHerzegovina, Spain and France. Forest Snow and Landscape Research 76: 391-396.

UL HAQUE, M. 2014. Identification, characterization and pathogenicity of Phytophthora spp. ASSOCIATED WITH THE MORTALITY OF Alnus glutinosa in Spain. 142.

VERMA, M., S.K. BRAR, R.D. TYAGI, R.Y. SURAMPALLI, and J.R. VALÉRO. 2007. Antagonistic fungi, Trichoderma spp.: Panoply of biological control. Biochemical Engineering Journal 37: 1-20.

VINALE， F., K. SIVASITHAMPARAM, E.L. GHISALBERTI, R. MARRA, S.L. WOO, and M. LORITO. 2008. Trichoderma-plant-pathogen interactions. Soil Biology and Biochemistry 40: $1-10$.

WAGNER BETTIOL, MARTA C. RIVERA, PEDRO MONDINO, JAIME R. MONTEALEGRE A., Y.C.C. 2014. Control biológico de enfermedades 
de plantas en América Latina y el Caribe. Facultad de Agricultura, Universidad de la República, Montevideo. $402 \mathrm{pp}$.

WEINDLING, R. 1932. Trichoderma lignorum as a parasite of other soil fungi. Phytopathology 22: 837-845.

WINGFIELD, M.J., A. HAMMERBACHER, R.J. GANLEY, E.T. STEENKAMP, T.R. GORDON, B.D. WINGFIELD, and T.A. COUTINHO. 2008. Pitch canker caused by Fusarium circinatum - A growing threat to pine plantations and forests worldwide. Australasian Plant Pathology 37: 319334.
YAKIMENKO, E.E., and I.D. GRODNITSKAYA. 2000. Effect of Trichoderma fungi on soil micromycetes that cause infectious conifer seedling lodging in Siberian tree nurseries. Microbiology 69: 726-729.

YANG. 1995. Phaeotheca dimorphospora as a potential biocontrol agent for shoot blight caused by Gremmeniella abietina. Canadian Journal Plant Pathology 17: 7-12.

ZABALGOGEAZCOA, I. 2008. Fungal endophytes and their interaction with plant pathogens. Spanish Journal of Agricultural Research 6: 138-146. 